\title{
Jurisdictional Etiquette Workgroup: An offshoot of a Syndromic Surveillance Consortium
}

\author{
Kike Oduba ${ }^{1}$, Tolulope Olumuyiwa ${ }^{1}$, Biru Yang ${ }^{1}$, Joanne Schulte ${ }^{1}$, Kaye Reynold ${ }^{2}$, Eric \\ Bakota 3 \\ ${ }^{1}$ Houston Health Department, Houston, Texas, United States, ${ }^{2}$ Fortbend County Health and Human Services, Richmond, Texas, United States, \\ ${ }^{3}$ Harris County Public Health, Houston, Texas, United States
}

Objective

- To demonstrate the importance of a cross-jurisdictional etiquette workgroup in the Texas Southeast region that leverages on the Syndromic Surveillance Consortium

- To promote data sharing and communicate the findings of disease to assist rapid investigation and data sharing

\section{Key words:}

ESSENCE (Electronic Surveillance System for Early notification of Community-based Epidemics)

Introduction

Syndromic data is shifting the way surveillance has been done traditionally. Most recently, surveillance has gone beyond city limits and county boundary lines. In southeast Texas, a regional consortium of public health agencies and stakeholders in the 13-County area governs the local ESSENCE system. The Houston Health Department, (HHD) is responsible for deploying ESSENCE to the entire region.

To effectively monitor the health of the region's population, a need arose to establish clear guidelines for disease investigation and data sharing triggered by syndromic surveillance across the area. Since Houston's instance of ESSENCE serves all 13 counties, the consortium instituted a cross- jurisdictional etiquette group. The purpose of the group is to determine the standard protocol for responding to ESSENCE alerts and best practices for data sharing and use among consortium members.

\section{Methods}

To achieve these goals, it was determined that a smaller group of stakeholders besides governing officials is needed to provide guidance for regional data sharing and use. The etiquette group was established in the first quarter of 2018 and it included four consortium representatives from the $6 / 5$ south region of Texas. Their first meeting tackled issues relating to data sharing.

\section{Results}

The following products emerged from the activities of the etiquette group within 3 months of its existence:

- Publication/presentation guidance/policy to avoid duplication of efforts and misrepresentation of jurisdiction.

- Procedure for alert responses

- Instructions for within-systems management of alerts;

- Instructions for events/times of interest (e.g., political convention, Olympics);

- Instructions of syndromes of interest/syndrome-specific policies;

- Instructions for changing the syndrome definition; 
- Notification procedures for identification of a single case of reportable disease/important free text element within data.

\section{Conclusions}

Cross jurisdictional workgroups can influence rapid investigations of disease, protect patient health information and promote privacy and data security and confidentiality by establishing set rules/guidelines for data exchange. All 13-counties in the region rely on these guidelines as a standard for responsibly accessing, using and sharing data in the Texas Southeast ESSENCE system.

Lessons Learned:

- As the etiquette group continues to evolve, there is need for more resources to help foster data use and sharing among jurisdictional partners.

- Partner engagement is limited due to ongoing process of configuring the new system ESSENCE.

- Since disease has no boundaries, allocation of jurisdictional responsibilities for responding to alerts should be operationalized

- Continuous training is essential to ensure all system users adhere to the protocols in place for meaningful data use and data sharing 